\title{
Apatinibascher Paper Apatinib-based targeted therapy against pulmonary sarcomatoid carcinoma: a case report and literature review
}

\author{
Xiaofeng $\mathrm{Li}^{1}$, Yueming $\mathrm{He}^{2}$, Jinfeng $\mathrm{Zhu}^{1}$, Hongxia Pang ${ }^{1}$, Yongwei Lin ${ }^{1}$ and Jinyang \\ Zheng ${ }^{3}$ \\ ${ }^{1}$ Department of Oncology, Affiliated Quanzhou First Hospital of Fujian Medical University, Quanzhou 362000, China \\ ${ }^{2}$ Department of Respiratory Diseases, Affiliated Quanzhou First Hospital of Fujian Medical University, Quanzhou 362000, \\ China \\ ${ }^{3}$ Department of Pathology, Affiliated Quanzhou First Hospital of Fujian Medical University, Quanzhou 362000, China \\ Correspondence to: Jinyang Zheng, email: Listar@tom.com \\ Keywords: angiogenesis; apatinib; sarcomatoid carcinoma; targeted therapy
}

Received: September 29, $2017 \quad$ Accepted: March 17, $2018 \quad$ Published: September 14, 2018

Copyright: Li et al. This is an open-access article distributed under the terms of the Creative Commons Attribution License 3.0 (CC BY 3.0), which permits unrestricted use, distribution, and reproduction in any medium, provided the original author and source are credited.

\section{ABSTRACT}

\begin{abstract}
Sarcomatoid carcinoma is a rare malignancy characterized by a combination of epithelial and sarcoma or sarcoma-like components. In this study, we reported one case of pulmonary sarcomatoid carcinoma and evaluated the safety and efficacy of apatinib, a tyrosine kinase inhibitor selectively targeting vascular endothelial growth factor receptor 2 , in treating this disease. The tumor mass was detected in the left lung of a 75-year-old man and showed positive immunostaining for cytokeratin (CK) 7, CK8, smooth muscle actin, CD31, and CD34. Next-generation sequencing analysis identified 4 mutations in NF1 (p.Q347Sfs*29), CDKN2A (p.G23V), ERBB3 (p.V104L), and TP53 (p.V157F) genes. The patient was given apatinib ( $250 \mathrm{mg}$ ) orally once per day. Sustained tumor regression was observed after apatinib treatment. There was no sever complication associated with apatinib therapy. In conclusion, apatinib-based targeted therapy may represent an important option for patients with sarcomatoid carcinoma.
\end{abstract}

\section{INTRODUCTION}

Sarcomatoid carcinoma is a rare form of cancer characterized by a combination of epithelial and sarcoma or sarcoma-like components [1]. Sarcomatoid carcinoma has been detected at multiple anatomic sites such as the prostate [2], lung [3], gallbladder [4], jejunum [5], and pelvic cavity [6]. Pulmonary sarcomatoid carcinoma accounts for $0.1-0.4 \%$ of all pulmonary cancers [7]. It has a highly metastatic property and exhibits a poor response to conventional chemotherapy $[8,9]$. Therefore, it is of significance in developing novel therapeutic strategies against this malignancy.

Apatinib is a tyrosine kinase inhibitor that selectively targets vascular endothelial growth factor receptor 2 (VEGFR2) expressed on tumor cells and shows a broad range of anticancer activity [10]. A randomized, double-blind, placebo-controlled phase III trial demonstrated that apatinib treatment resulted in a significant increase in median overall survival (6.5 vs. 4.7 months) and progression-free survival (2.6 vs. 1.8 months) in patients with chemotherapy-refractory advanced gastric cancer, compared to the placebo group [11]. A single-center randomized controlled trial showed that the combination of transcatheter arterial chemoembolization (TACE) with apatinib conferred survival benefits to patients with advanced hepatocellular carcinoma (HCC) [12]. In addition, several studies involving individual cases have documented the anticancer activity of apatinib in patients with advanced disease $[13,14]$. 
In this report, we described one case of sarcomatoid carcinoma in the lung and showed the therapeutic effect of apatinib against this malignancy.

\section{CASE REPORT}

A 75-year-old man was admitted to our hospital on June 15, 2016 with a chief complaint of coughing and blood-stained sputum for over 1 month. He presented chest stuffiness and shortness of breath and required continuous oxygen inhalation. The patient also presented coronary arteriosclerosis. There were no symptoms of dizziness, chest pain, fever, and vomiting. He had a smoking history of over 60 years and smoked up to 30 cigarettes a day. Tumor markers carcinoembryonic antigen and neuronspecific enolase were within the normal range. Chest computed tomography $(\mathrm{CT})$ revealed a soft mass $(63 \times 48$ $\mathrm{mm}$ ) and signs of pneumonia in the left lung (Figure 1A). Swollen lymph nodes above the left clavicle were evident on color Doppler ultrasound images. CT-guided lung biopsy was conducted. The mass was histologically diagnosed as sarcomatoid carcinoma, which was staged as IV according to the 8th edition of the AJCC/UICC TNM staging system for lung cancer. Supraclavicular lymph node metastasis was detected. Immunohistochemistry showed that tumor cells were positive for cytokeratin (CK) 7, CK8, and thyroid transcription factor 1 (TTF-1) and negative for CK5/6, p63, p40, CD56, and synaptophysin.
Of note, the tumor was abundantly vascular, with strong immunostaining for smooth muscle actin (SMA), CD31, and CD34 (Figure 2). As determined by next-generation sequencing, this patient displayed 4 mutations in $N F 1$ (p.Q347Sfs"29), CDKN2A (p.G23V), ERBB3 (p.V104L), and TP53 (p.V157F) genes (Table 1).

The patient had an ECOG performance status of 3 and thus was precluded from chemotherapy. After receiving informed consent, he was given apatinib (250 $\mathrm{mg}$ ) orally once per day from June 27, 2016. Haemoptysis symptoms disappeared and chest stuffiness was relieved 5 days after apatinib treatment. At 10 days after treatment, he restored normal breathing. Follow-up CT at 1 (Figure 1B), 3 (Figure 1C), and 9 (Figure 1D) months showed that the tumor regressed to $54 \times 43 \mathrm{~mm}, 41 \times 40 \mathrm{~mm}$, and $36 \times$ $28 \mathrm{~mm}$, respectively. Until drafting the manuscript (14 months after therapy), tumor regression was observed. There was no evident complication associated with apatinib therapy.

\section{DISCUSSION}

Clinical detection of sarcomatoid carcinoma is a rare event [1-3]. In this study, we reported a case of sarcomatoid carcinoma occurring in the lung. Compared to other types of lung cancer, pulmonary sarcomatoid carcinoma has a worse overall survival [15]. Pulmonary sarcomatoid carcinoma is characterized by frequent
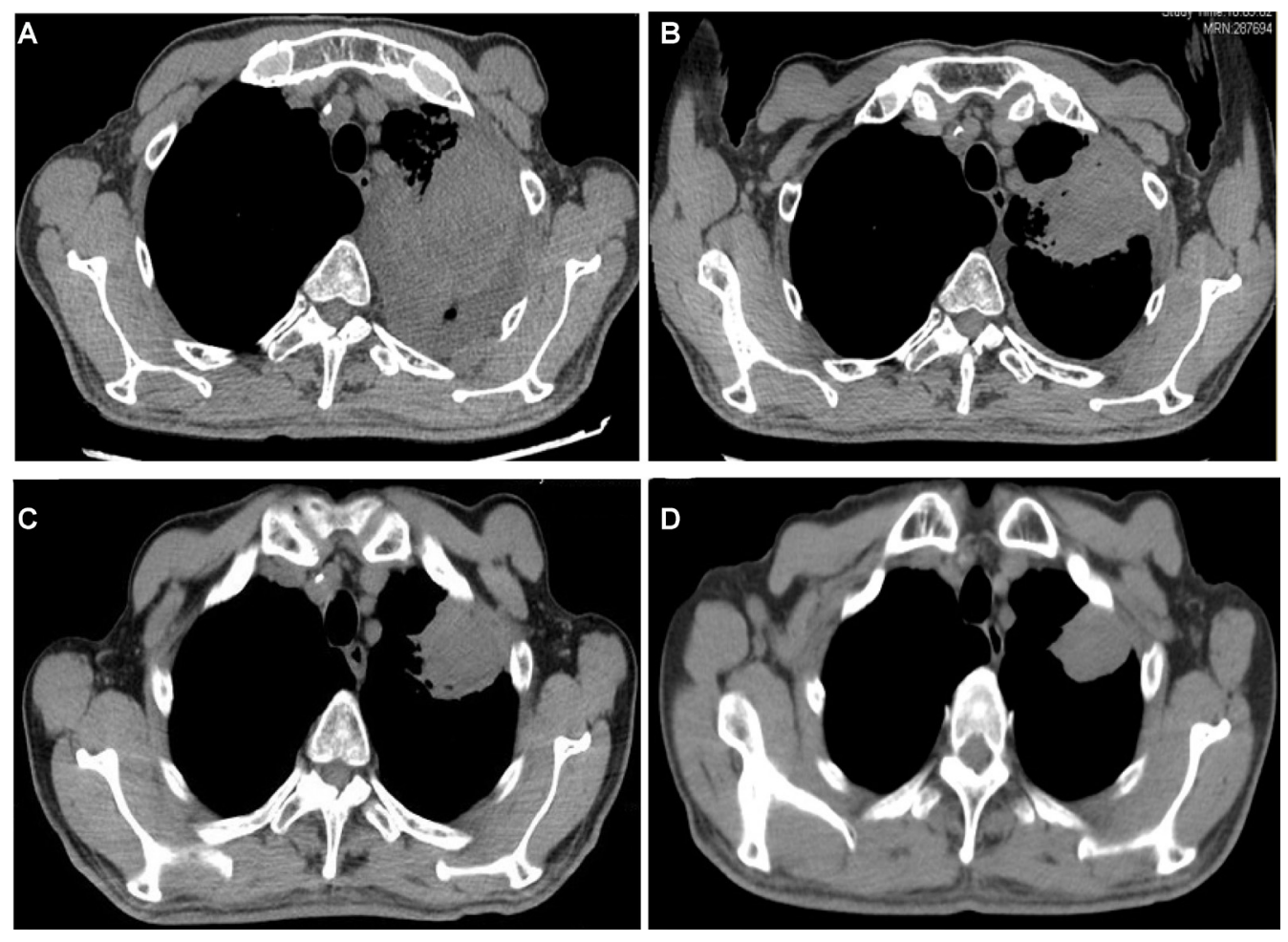

Figure 1: Chest CT showed tumor regression in the left lung after apatinib therapy. (A-D) before and 1, 3, and 9 months after treatment, respectively. 
Table 1: Detection of mutations in the patient with pulmonary sarcomatoid carcinoma by next-generation sequencing

\begin{tabular}{lcccc}
\hline Gene & Chromosomal location & Reference sequence & Nucleotide change & Amino acid change \\
\hline NF1 & chr17:29527590 & NM_001042492 & c.1039del & p.Q347Sfs*29 \\
CDKN2A & chr9:21974759 & NM_000077 & c.G68T & p.G23V \\
TP53 & chr17:7578461 & NM_001126112 & c.G469T & p.V157F \\
ERBB3 & chr12:56478854 & NM_001982 & c.G310T & p.V104L \\
\hline
\end{tabular}

gene mutations. Li et al. [3] performed next-generation sequencing in 7 patients with pulmonary sarcomatoid carcinoma and identified 136 putative somatic variants and one gene fusion. Terra et al. [16] analyzed 33 cases of pulmonary sarcomatoid carcinoma by next-generation sequencing and found that $72 \%$ had at least one genetic variant, 58\% TP53 mutations, and 30\% KRAS mutations. Similarly, Lococo et al. [17] reported that 39 of the 49 pulmonary sarcomatoid carcinomas (80\%) carried at least one mutation. Consistent with the previous studies, we also identified TP53 mutation in this case. Of note, we found novel mutations in $N F 1, C D K N 2 A$, and $E R B B 3$ genes. NF1 acts as a tumor suppressor and its loss-offunction mutation results in activation of the RAS/RAF/ MAPK signaling pathway, consequently contributing to tumor progression $[18,19]$. In this study, we showed that the mutation (c.1039del) led to the glutamine to serine change at position 347 and introduction of a stop codon, which should terminate protein translation prematurely. $C D K N 2 A$ mutation is also linked to development of cancer [20, 21]. It has been reported that $C D K N 2 A$ p.Arg112dup mutation carriers have an elevated risk for pancreatic, lung, head and neck and gastro-oesophageal carcinomas [21]. ERBB3 is implicated in multiple aspects of tumor biology [22, 23]. ERBB3 mutations increase the response to afatinib in platinum-refractory metastatic urothelial carcinoma [22]. Taken together, it is suggested that genetic mutations, in particular in TP53, NF1, CDKN2A, and $E R B B 3$ genes may contribute to the pathogenesis of sarcomatoid carcinoma.

Due to poor ECOG performance status, the patient reported in this study did not receive chemotherapy. CD31 and CD34 immunostaining analysis showed remarkably abundant microvessels in the tumor mass of our patient, indicating active tumor angiogenesis. A previous study has provided evidence that mutation in $N F 1$ gene can augment
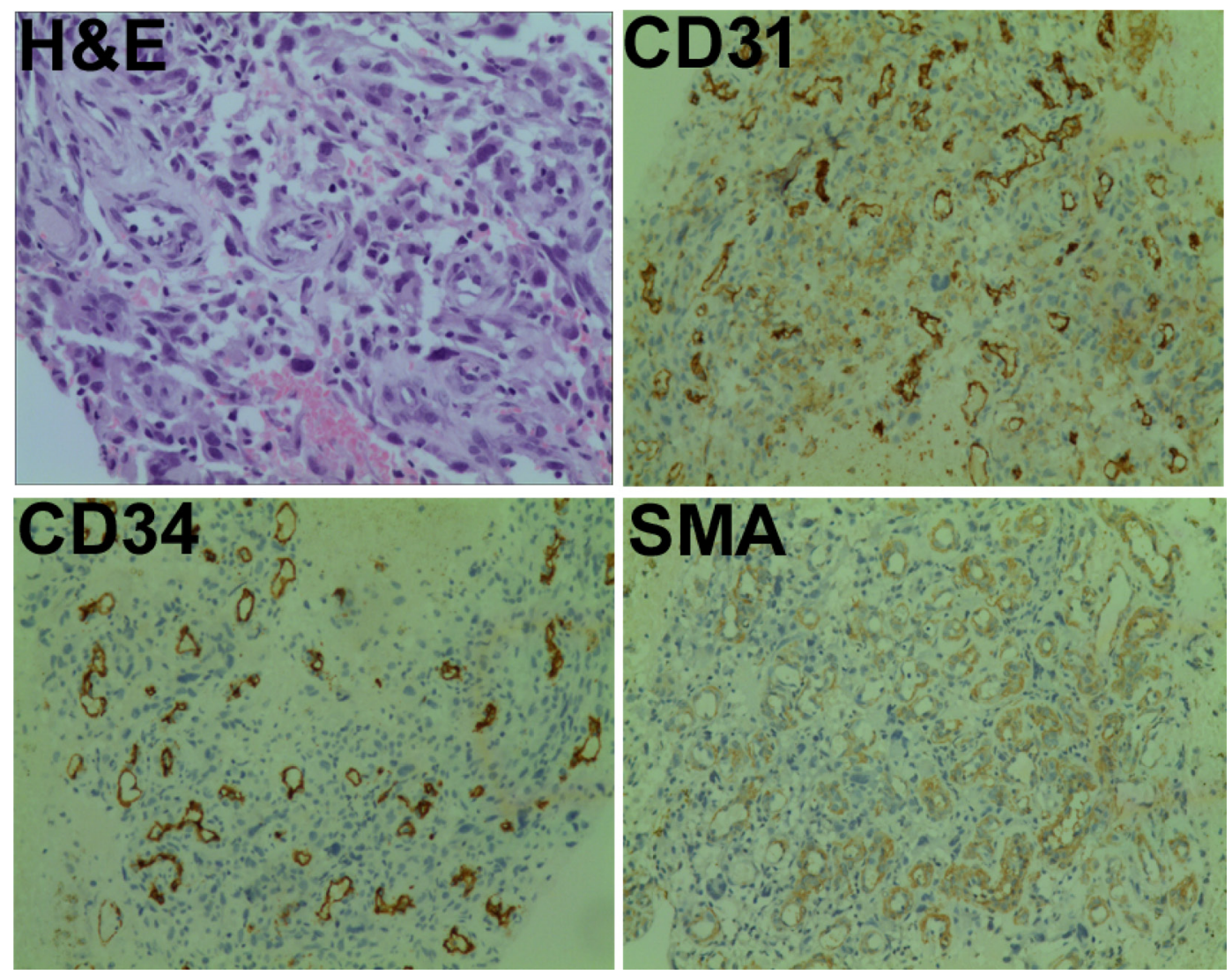

Figure 2: Pathological findings. Tumor samples were subjected to hematoxylin-eosin (H\&E) staining or immunostained for smooth muscle actin (SMA), CD31, and CD34. Magnification: $\times 200$. 
angiogenesis in a mouse model by promoting endothelial cell proliferation and migration [24]. p53 functional deficiency was reported to enhance fibroblast-mediated angiogenesis in colon cancer [25]. TP53 mutations are associated with the sensitivity to VEGF/VEGFR inhibitors [26]. TP53 mutational status has been identified as a predictive biomarker of response to VEGFR inhibitors in advanced sarcoma [27]. These studies suggest that the substantial tumor angiogenesis in the present case may be a result of mutations in multiple genes including TP53 and NF1.

Given the presence of active tumor angiogenesis, we sought to control disease progression in the patient by targeting pro-angiogenic signaling. Apatinib, as a tyrosine kinase inhibitor targeting VEGFR2, has exhibited anticancer activity on many cancer types $[10,11,28]$. Coadministration of apatinib has been reported to augment antitumor activity of gefitinib on non-small cell lung cancer with resistance to epidermal growth factor receptor tyrosine kinase inhibitors [14]. Zhang et al. [29] reported that apatinib leads to partial or complete response in 2 cases of refractory recurrent malignant gliomas. Apatinib can improve progression-free survival and overall survival in patients with chemotherapy-refractory advanced metastatic gastric cancer [30]. Deng et al. [31] described that a case with for chemotherapy-refractory advanced epithelial ovarian cancer obtained survival benefits from apatinib treatment. In the present study, sustained apatinib treatment caused a marked tumor regression, without producing evident toxicities. The good response observed may be ascribed to suppression of tumor angiogenesis caused by mutation of specific key genes. In support of this hypothesis, it has been documented that apatinib therapy can evoke stable disease response in advanced lung adenocarcinoma patients with KRAS mutation [13]. However, the exact mechanism governing apatinib response needs to be further clarified.

In summary, our present case confirms frequent genetic mutations occurring in pulmonary sarcomatoid carcinoma. To our best of knowledge, this is the first report of the successful treatment of pulmonary sarcomatoid carcinoma with apatinib. Prospective studies are warranted to validate the therapeutic efficacy of apatinib in sarcomatoid carcinomas.

\section{CONFLICTS OF INTEREST}

None.

\section{REFERENCES}

1. Mark D, Boyd C, Eatock F. Adrenal sarcomatoid carcinoma: a case report and review of the literature. Ulster Med J. 2014; 83:89-92.

2. Markowski MC, Eisenberger MA, Zahurak M, Epstein JI, Paller CJ. Sarcomatoid Carcinoma of the Prostate:
Retrospective Review of a Case Series From the Johns Hopkins Hospital. Urology. 2015; 86:539-43.

3. Li X, Wang D, Zhao Q, Ren D, Ren F, Chen G, Liu H, Chen J. Clinical Significance and Next-Generation Sequencing of Chinese Pulmonary Sarcomatoid Carcinoma. Sci Rep. 2017; 7:3947.

4. Feng F, Cheng Q, Yang L, Zhang D, Ji S, Zhang Q, Lin Y, Li F, Xiong L, Liu C, Jiang X. Guidance to rational use of pharmaceuticals in gallbladder sarcomatoid carcinoma using patient-derived cancer cells and whole exome sequencing. Oncotarget. 2017; 8:5349-60. https://doi. org/10.18632/oncotarget.14146.

5. Kwok CM. Sarcomatoid carcinoma of the jejunum with gastric metastases: A case report and review of the literature. Int J Surg Case Rep. 2016; 28:161-4.

6. Fang C, Fan CW, Yu YY, Wang C, Yang L, Li Y, Mo XM, Zhou ZG. Severe Hypoglycemia Caused by Recurrent Sarcomatoid Carcinoma in the Pelvic Cavity: A Case Report. Medicine (Baltimore). 2015; 94:e1577.

7. Park JS, Lee Y, Han J, Kim HK, Choi YS, Kim J, Shim YM, Kim K. Clinicopathologic outcomes of curative resection for sarcomatoid carcinoma of the lung. Oncology. 2011; 81:206-13.

8. Yendamuri S, Caty L, Pine M, Adem S, Bogner P, Miller A, Demmy TL, Groman A, Reid M. Outcomes of sarcomatoid carcinoma of the lung: a Surveillance, Epidemiology, and End Results Database analysis. Surgery. 2012; 152:397-402.

9. Vieira T, Girard N, Ung M, Monnet I, Cazes A, Bonnette P, Duruisseaux M, Mazieres J, Antoine M, Cadranel J, Wislez M. Efficacy of first-line chemotherapy in patients with advanced lung sarcomatoid carcinoma. J Thorac Oncol. 2013; 8:1574-7.

10. de Mello RA, de Oliveira J, Antoniou G. Angiogenesis and apatinib: a new hope for patients with advanced gastric cancer? Future Oncol. 2017; 13:295-8.

11. Li J, Qin S, Xu J, Xiong J, Wu C, Bai Y, Liu W, Tong J, Liu Y, Xu R, Wang Z, Wang Q, Ouyang X, et al. Randomized, Double-Blind, Placebo-Controlled Phase III Trial of Apatinib in Patients With ChemotherapyRefractory Advanced or Metastatic Adenocarcinoma of the Stomach or Gastroesophageal Junction. J Clin Oncol. 2016; 34:1448-54.

12. Lu W, Jin XL, Yang C, Du P, Jiang FQ, Ma JP, Yang J, Xie P, Zhang Z. Comparison of efficacy between TACE combined with apatinib and TACE alone in the treatment of intermediate and advanced hepatocellular carcinoma: A single-center randomized controlled trial. Cancer Biol Ther. 2017; 18:433-8.

13. Zeng DX, Wang CG, Huang JA, Jiang JH. Apatinib in the treatment of advanced lung adenocarcinoma with KRAS mutation. Onco Targets Ther. 2017; 10:4269-72.

14. Li F, Zhu T, Cao B, Wang J, Liang L. Apatinib enhances antitumour activity of EGFR-TKIs in non-small cell lung 
cancer with EGFR-TKI resistance. Eur J Cancer. 2017; 84:184-92.

15. Roesel C, Terjung S, Weinreich G, Hager T, Chalvatzoulis E, Metzenmacher M, Welter S. Sarcomatoid carcinoma of the lung: a rare histological subtype of non-small cell lung cancer with a poor prognosis even at earlier tumour stages. Interact Cardiovasc Thorac Surg. 2017; 24:407-13.

16. Terra SB, Jang JS, Bi L, Kipp BR, Jen J, Yi ES, Boland JM. Molecular characterization of pulmonary sarcomatoid carcinoma: analysis of 33 cases. Mod Pathol. 2016; 29:824-31.

17. Lococo F, Gandolfi G, Rossi G, Pinto C, Rapicetta C, Cavazza A, Cesario A, Galeone C, Paci M, Ciarrocchi A. Deep Sequencing Analysis Reveals That KRAS Mutation Is a Marker of Poor Prognosis in Patients with Pulmonary Sarcomatoid Carcinoma. J Thorac Oncol. 2016; 11:1282-92.

18. Philpott C, Tovell H, Frayling IM, Cooper DN, Upadhyaya M. The NF1 somatic mutational landscape in sporadic human cancers. Hum Genomics. 2017; 11:13.

19. Pemov A, Li H, Patidar R, Hansen NF, Sindiri S, Hartley SW, Wei JS, Elkahloun A, Chandrasekharappa SC, Boland JF, Bass S, Mullikin JC, Khan J, et al; NISC Comparative Sequencing Program; NCI DCEG Cancer Genomics Research Laboratory. The primacy of NF1 loss as the driver of tumorigenesis in neurofibromatosis type 1-associated plexiform neurofibromas. Oncogene. 2017; 36:3168-77.

20. Larque AB, Conde L, Hakim S, Alos L, Jares P, Vilaseca I, Cardesa A, Nadal A. P16(INK ${ }^{4}$ a) overexpression is associated with CDKN2A mutation and worse prognosis in HPVnegative laryngeal squamous cell carcinomas. Virchows Arch. 2015; 466:375-82.

21. Helgadottir H, Höiom V, Jönsson G, Tuominen R, Ingvar C, Borg A, Olsson H, Hansson J. High risk of tobacco-related cancers in CDKN2A mutation-positive melanoma families. J Med Genet. 2014; 51:545-52.

22. Choudhury NJ, Campanile A, Antic T, Yap KL, Fitzpatrick CA, Wade JL 3rd, Karrison T, Stadler WM, Nakamura Y, O'Donnell PH. Afatinib Activity in Platinum-Refractory Metastatic Urothelial Carcinoma in Patients With ERBB Alterations. J Clin Oncol. 2016; 34:2165-71.
23. Dahlhoff M, Schäfer M, Muzumdar S, Rose C, Schneider MR. ERBB3 is required for tumor promotion in a mouse model of skin carcinogenesis. Mol Oncol. 2015; 9:1825-33.

24. Wu M, Wallace MR, Muir D. Nf1 haploinsufficiency augments angiogenesis. Oncogene. 2006; 25:2297-303.

25. Hayashi Y, Tsujii M, Kodama T, Akasaka T, Kondo J, Hikita H, Inoue T, Tsujii Y, Maekawa A, Yoshii S, Shinzaki $\mathrm{S}$, Watabe K, Tomita Y, et al. p53 functional deficiency in human colon cancer cells promotes fibroblast-mediated angiogenesis and tumor growth. Carcinogenesis. 2016; 37:972-84.

26. Wheler JJ, Janku F, Naing A, Li Y, Stephen B, Zinner R, Subbiah V, Fu S, Karp D, Falchook GS, Tsimberidou AM, Piha-Paul S, Anderson R, et al. TP53 Alterations Correlate with Response to VEGF/VEGFR Inhibitors: Implications for Targeted Therapeutics. Mol Cancer Ther. 2016; 15:2475-85.

27. Koehler K, Liebner D, Chen JL. TP53 mutational status is predictive of pazopanib response in advanced sarcomas. Ann Oncol. 2016; 27:539-43.

28. Peng QX, Han YW, Zhang YL, Hu J, Fan J, Fu SZ, Xu $\mathrm{S}$, Wan Q. Apatinib inhibits VEGFR-2 and angiogenesis in an in vivo murine model of nasopharyngeal carcinoma. Oncotarget. 2017; 8:52813-22. https://doi.org/10.18632/ oncotarget.17264.

29. Zhang H, Chen F, Wang Z, Wu S. Successful treatment with apatinib for refractory recurrent malignant gliomas: a case series. Onco Targets Ther. 2017; 10:837-45.

30. Li J, Qin S, Xu J, Guo W, Xiong J, Bai Y, Sun G, Yang Y, Wang L, Xu N, Cheng Y, Wang Z, Zheng L, et al. Apatinib for chemotherapy-refractory advanced metastatic gastric cancer: results from a randomized, placebo-controlled, parallel-arm, phase II trial. J Clin Oncol. 2013; 31:3219-25.

31. Deng L, Wang Y, Lu W, Liu Q, Wu J, Jin J. Apatinib treatment combined with chemotherapy for advanced epithelial ovarian cancer: a case report. Onco Targets Ther. 2017; 10:1521-5. 